\title{
Ghazaleh Nassibi* \\ Das Verbot sittenwidriger Löhne und die Europäische Sozialcharta
}

\section{Einleitung}

Die neue Bundesregierung hat sich im Koalitionsvertrag darauf geeinigt, „die Rechtsprechung zum Verbot sittenwidriger Löhne gesetzlich festzuschreiben, um Lohndumping zu verhindern". ${ }^{1}$ Auf den ersten Blick erscheint dies unterstützenswert, denn der Schutz von Beschäftigten vor Niedriglöhnen ist angesichts der immer weiteren Zunahme prekärer Beschäftigungsverhältnisse und niedrig entlohnter Tätigkeiten ${ }^{2}$ dringend geboten. Der folgende Artikel untersucht - nach einem Überblick über die verschiedenen Wege der Lohnfestsetzung in Deutschland -, was unter „Rechtsprechung zum Verbot sittenwidriger Löhne“ $\mathrm{zu}$ verstehen ist und wie weit ihr Schutz reicht. Zudem soll geklärt werden, inwieweit auch die Europäische Sozialcharta (ESC) in Deutschland zum Schutz vor Niedriglöhnen herangezogen werden kann.

\section{Schutz vor sittenwidrigen Löhnen}

\section{Festsetzung der Lobnböbe in Deutschland}

Im Gegensatz zu den meisten anderen europäischen Ländern gibt es in Deutschland keinen einheitlichen gesetzlichen Mindestlohn. ${ }^{3}$ Unter der neuen schwarzgelben Regierung ist die Einführung eines solchen auch nicht zu erwarten. ${ }^{4}$ Vielmehr obliegt hier die Festsetzung der Lohnhöhe im Rahmen der Regelungskompetenz nach Art. 9 Abs. 3 GG zum größten Teil den Tarifvertragsparteien, die dazu Tarifverträge abschließen. Denn die Sozialpartner können die Arbeitsbedingungen am besten in eigener Verantwortung und ohne staatlichen Einfluss regeln. ${ }^{5}$ Seit Mitte der 90er Jahre ist jedoch sowohl ein genereller Rückgang der Tarifbindung als auch eine Erosion des Flächentarifvertrags zu verzeichnen. ${ }^{6}$ In tariffreien Erwerbsbereichen, wo Niedriglöhne besonders häufig zu finden sind, kommen Tarifverträge nur dann zur Anwendung, wenn sie für allgemeinverbindlich erklärt werden. ${ }^{7}$ Die Zahl allgemeinverbindlich erklärter Tarifverträge ist in den letzten Jahren aber stetig zurückgegangen. ${ }^{8}$ Allgemeinverbindlich erklärte Entgelttarifverträge bildeten zudem schon immer den kleineren Anteil

* Für Anregungen und Hinweise danke ich Eva Kocher, Andreas Fischer-Lescano und Olaf Deinert.

1 Koalitionsvertrag zwischen CDU, CSU und FDP, 17. Legislaturperiode, S. 13: http://www.spiegel.de/ media/0,4906,21958,00.pdf (abgerufen am 2.11.2009).

2 Vgl. dazu Kalina/Weinkopf, IAQ-Report 5/2009, S. 1; Bispinck/Schulten, WSI-Mitteilungen 2009, 202.

3 Zur aktuellen Entwicklung der nationalen Lohnpolitiken in der EU: Schulten, WSI-Mitteilungen 2009, $154 \mathrm{f}$.

4 Vgl. Koalitionsvertrag zwischen CDU, CSU und FDP (Fn. 1), S. 13.

5 Strybny, FS für Leinemann, 2006, S. 796; Peter, in: Peter/Kempen/Zachert, Die Sicherung tariflicher Mindeststandards, 2004, S. 104.

6 Bispinck/Schulten (Fn. 2), S. 201; Dieterich, KJ 2008, 71.

7 Durch die Allgemeinverbindlichkeit von Tarifverträgen werden die von den Tarifvertragsparteien verhandelten tariflichen Mindestarbeitsbedingungen auf die nicht tarifgebundenen Arbeitsverhältnisse des tariflichen Geltungsbereichs erstreckt.

8 Däubler/Lakies, TVG-Kommentar, 2. Aufl. 2006, $\$ 5$, Anh. 1, Rn. $13 \mathrm{ff}$. 
daran. ${ }^{9}$ In einigen regionalen Tarifbereichen gibt es allgemeinverbindliche Entgelttarifverträge nach dem Tarifvertragsgesetz (TVG). ${ }^{10}$ Daneben gibt es noch die Branchen, in denen über das Arbeitnehmerentsendegesetz (AEntG) oder das Mindestarbeitsbedingungengesetz (MindArbBG) Löhne für allgemeinverbindlich erklärt werden können. Damit zeigt der Staat, dass er die besondere Schutzbedürftigkeit von Beschäftigten vor Niedriglöhnen anerkennt. Er geht aber nur dort spezifisch tarifersetzend vor, wo es keine funktionsfähigen Tarifvertragsparteien und eine nur geringe Tarifbindung gibt, so mit dem MindArbBG. Ansonsten beschränkt er sich darauf, die in Tarifverträgen vereinbarten Arbeitsbedingungen durch staatlichen Gestaltungsbefehl zu erstrecken, so in Branchen mit hoher Tarifbindung durch $\ 5$ TVG und durch das AEntG. In der öffentlichen Auftragsvergabe kann schließlich die sog. Tariftreueerklärung zu einer indirekten staatlichen Entgeltkontrolle führen.

Das MindArbBG und das AEntG wurden in der letzten Legislaturperiode nach langen Debatten reformiert. ${ }^{11}$ Doch werden die Reformen vielfach kritisiert, da die Gesetze nach wie vor Lücken aufweisen: Über das AEntG können nur in Branchen mit hoher Tarifbindung bestehende Tarifverträge für die gesamte Branche für allgemeinverbindlich erklärt werden. Dort aber, wo keine Tarifverträge existieren oder nur eine geringe Tarifbindung besteht, entfaltet das Gesetz keine Wirkung. ${ }^{12} \mathrm{Ob}$ das MindArbBG zu einem wirksamen Instrument gegen Lohndumping wird, hängt u.a. davon ab, ob mit dem Gesetz auch über das Existenzminimum hinausgehende, angemessene Löhne festgesetzt werden. ${ }^{13}$ Auch mit $₫ 5$ TVG sind keine strukturellen Veränderungen durchsetzbar, da damit nur eine punktuelle Ausweitung von Tarifbedingungen erreicht, nicht aber die durch Deregulierung des Flächentarifvertrags verlorengegangene Geltungskraft des Tarifvertrags zurückgewonnen werden kann. ${ }^{14}$ Schließlich bewirken Tariftreueklauseln in der öffentlichen Auftragsvergabe zwar die Sicherung von Mindestlohnstandards, aber sie erfassen auch „nur“ den Bereich der öffentlichen Vergabe. Zudem hat das Rüffert-Urteil des EuGH vom 3.4.2008 große Unsicherheit über die Zulässigkeit von Tariftreueklauseln in den Vergabegesetzen der Bundesländer hervorgerufen. ${ }^{15}$

Abgesehen davon gibt es in Deutschland keinen für alle Branchen verbindlichen Mindestlohn. So ist bei vielen Arbeitsverhältnissen die Lohnhöhe weder tariflich noch gesetzlich geregelt. Hier gilt die Privatautonomie, die prinzipiell die Vereinbarung jeder Lohnhöhe ermöglicht. ${ }^{16}$ Dabei wird von der Richtigkeitsgewähr vertraglicher Vereinbarungen ausgegangen. ${ }^{17}$ Wenn aber zwischen den Vertragsparteien ein offensichtliches strukturelles Ungleichgewicht besteht, ist die Ordnungsfunktion des Privatrechts gestört. ${ }^{18}$ Insbesondere beim Abschluss von Arbeitsverträgen ist ein strukturelles Ungleichgewicht gegeben, weil sich die Beschäftigten, die auf den Verkauf ihrer Arbeitskraft angewiesen sind, sich typi-

10 Bispinck/Schulten WSI-Mitteilungen 2008, 152; Erfurter Kommentar/Franzen, 10. Aufl. 2010, \5 TVG Rn. 3.

11 Eingehend zu beiden Reformen: Sittard, NZA 2009, 346.

12 Bispinck/Schulten (Fn. 10), S. 153 f.; Schulten (Fn. 3), S. 150.

13 Vgl. zur Reform des MindArbBG näher: Sansone/Ulber, AuR 2008, 129; Bayreuther, NJW 2009, 2011.

14 Zachert, WSI-Mitteilungen 2003, 418; vgl. Peter, Gesetzlicher Mindestlohn, 1995, S. 90.

15 EuGH 3.4.2008, Rs. C-346/06; vgl. dazu aus der Literatur: z.B. Kocher, DB 2008, 1042; Dräger/Mileva, Sozialismus 2008, 28; Becker, JZ 2008, 891; Koberski/Schierle, RdA 2008, 233; Wolter, AuR 2006, 137; Dobmann, VergabeR 3/2008, 484; Bayreuther, NZA 2008, 626; Wittjen, ZfBR 2009, 30.

16 Lakies, NZA-RR 2002, 339; Preis, AuR 1994, 141; Fastrich, RdA 1997, 67 f.

17 Zum Gedanken der „Richtigkeitsgewähr“, der auf Schmidt-Rimpler zurückgeht: vgl. Preis (Fn. 17), S. 141.

18 Bayreuther (Fn. 9), S. 2023 f.; Fastrich (Fn. 16), S. 68. 
scherweise in einer unterlegenen Verhandlungsposition befinden. ${ }^{19}$ Denn Arbeitnehmer tauschen nicht nur - wie im sonstigen Zivilrecht - irgendeine Leistung aus, sondern treten als Person mit dem Arbeitsvertrag in ein vom Arbeitgeber durch das Direktionsrecht bestimmtes Herrschaftsverhältnis ein. ${ }^{20}$ In solchen Fällen hat das Bundesverfassungsgericht der Privatautonomie Grenzen gezogen und eine Inhaltskontrolle am Maßstab der Generalklauseln gefordert. ${ }^{21}$ Hauptansatzpunkt ist hierbei der sittenwidrige Lohnwucher nach $\$ 138$ BGB.

\section{Sittenwidriger Lobnwucher nach $\int 138 B G B$}

Nach $\ 138$ Abs. 1 BGB ist ein Rechtsgeschäft nichtig, das gegen die guten Sitten verstößt. Abs. 2 verbietet als speziellen Fall der Sittenwidrigkeit das wucherische Rechtsgeschäft.

Abs. 2 ist erfüllt, wenn eine Lohnvereinbarung unter Ausbeutung der Zwangslage, der Unerfahrenheit, dem Mangel an Urteilsvermögen oder der erheblichen Willensschwäche des Beschäftigten zustande kommt und wenn sie ein auffälliges Missverhältnis zwischen dem Wert der Arbeitsleistung und Lohn aufweist. ${ }^{22} \mathrm{Da}$ neben muss auch die subjektive Tatbestandsseite erfüllt sein. Nur wenn Abs. 2 abgelehnt wird, kommt Abs. 1 zum Zug. Auch hier wird ein auffälliges Missverhältnis verlangt. ${ }^{23}$ Zusätzlich sind aber noch besondere Sittenwidrigkeitsumstände erforderlich, ${ }^{24}$ so z.B. eine „verwerfliche Gesinnung des Arbeitgebers“ 25 oder die Missachtung schutzwürdiger Interessen der Allgemeinheit oder Dritter. ${ }^{26}$ Aus subjektiver Sicht liegt die Sittenwidrigkeit bei Abs. 1 darin, dass der überlegene Teil die schwächere Lage des anderen Teils bewusst zu seinem Vorteil ausnutzt oder sich böswillig oder leichtfertig der Erkenntnis verschließt, dass sich der andere Teil nur unter dem Zwang der Verhältnisse auf den ungünstigen Vertrag einlässt. ${ }^{27}$

\subsection{Bezugswert für das auffällige Missverhältnis}

Wichtig ist also die Bestimmung des auffälligen Missverbältnisses zwischen Arbeitsleistung und Lohn. Dafür ist ein Maßstab erforderlich, an dem die zu untersuchenden Löhne gemessen werden können (= Bezugswert). ${ }^{28}$ Im Fokus steht dabei die Hauptleistungspflicht der Beschäftigten zur Arbeitsleistung im Gegenzug zur Lohnzahlungspflicht der Arbeitgeber/innen. ${ }^{29}$

Das BAG meint, die Arbeitsleistung von Beschäftigten sei nach ihrem objektiven Wert zu beurteilen, der sich nach der verkehrsüblichen Vergütung richte. ${ }^{30}$ Zur Ermittlung der verkehrsüblichen Vergütung sei nicht nur auf den Vergleich mit den Tariflöhnen des Wirtschaftszweiges abzustellen, sondern auch auf das all-

BAG, NZA 1994, 939 f.; BVerfG, AuR 1992, 189; BVerfG, NZA 2007, 85; Lakies (Fn. 16), S. 340; Reinecke, NZA-Sonderbeilage 3/2000, 25; Dieterich, RdA 1995, 135; kritisch zur These von der gestörten Vertragsparität: Rieble, Arbeitsmarkt und Wettbewerb, 1996, Rn. 847 f., 926.

20 Zachert, RdA 2001, 106 (mit Verweis auf Gedanken des Arbeitsrechtlers und Rechtssoziologen H. Sinzheimer).

21 BVerfGE 81, 242 (254f.); BVerfGE 89, 214 (232f.); BVerfG, NJW 1994, 2750; BVerfG, ZIP 1996, 957.

22 BGH, NJW 2003, 1860 f.; BAG, DB 2004, $1432 \mathrm{f}$.

23 BAG, AuR 2001, 510; Lakies (Fn. 17), S. 340.

24 BGHZ 146, 298; BGH, NJW 1999, 3190; BGH, NJW 2002, 56.

25 BAG, AP Nr. 30 zu $₫ 138$ BGB; BAG, AP Nr. 1 zu $\$ 138$ BGB.

26 BGHZ 20, 43 (50 f.); BGH, DB 1976, 573.

27 BAG, AP Nr. 37 zu $\ 138$ BGB; BAG, AP Nr. 39 zu $\$ 138$ BGB; BGH, NJW 2001, 1127.

28 Vgl. Peter, AuR 2001, 511; Lakies (Fn. 16), S. 341.

29 Erfurter Kommentar/Preis, 10. Aufl. 2010, \$612 BGB, Rn. 639.

30 BAG, NZA 2009, 837; BAG, DB 2004, 1432; BAG, AuR 2001, 510. 
gemeine Lohnniveau im Wirtschaftsgebiet. ${ }^{31}$ Wenn aber in einem Wirtschafts-

gebiet üblicherweise Tariflohn gezahlt werde, sei davon auszugehen, dass der Tariflohn den Wert der Arbeitsleistung bestimme. ${ }^{32}$ Wenn der übliche Lohn im Wirtschaftsgebiet unterhalb des Tariflohns liege, dann bestimme das allgemeine Lohnniveau im Wirtschaftsgebiet den Wert der Arbeitsleistung. ${ }^{33}$ Dies hat das BAG nun auch in seinem jüngsten Lohnwucher-Urteil vom 22. April 2009 bekräftigt. ${ }^{34}$ Die unteren Instanzen der Arbeitsgerichte und die Literatur orientieren sich zumeist am Tariflohn. ${ }^{35}$

\subsection{Grenzwert für das auffällige Missverbältnis}

Neben dem Bezugswert ist auch der sog. Grenzwert erforderlich, der bestimmt, ab welchem Prozentwert eine vom Bezugswert abweichende Bezahlung sittenwidrig ist. ${ }^{36}$ Das Gesetz lässt diese Frage offen.

So gab es dazu in der Vergangenheit bisher verschiedene Urteile des BAG. ${ }^{37}$ Erst mit seinem jüngsten Lohnwucher-Urteil hat das BAG einen klaren Grenzwert für sittenwidrige Löhne bestimmt und festgelegt, dass ein auffälliges Missverhältnis zwischen Leistung und Gegenleistung vorliegt, wenn der Lohn nicht einmal 2/3 eines in der Branche oder Wirtschaftsregion üblichen Tariflohns erreicht. ${ }^{38}$ Damit folgt es erstmals ausdrücklich der Entscheidung des BGH von 1997 zur Strafbarkeit des Lohnwuchers nach $\$ 302$ a StGB a.F. (\$ 291 StGB n.F.). ${ }^{39}$ An diesem Urteil orientieren sich gerade auch in jüngster Zeit viele Arbeitsgerichte. ${ }^{40}$ Daneben entscheiden die Arbeitsgerichte aber auch immer wieder Fälle mit abweichenden Grenzwerten. ${ }^{41}$ Interessant ist dabei ein Urteil des Arbeitsgerichts Bremen, wonach eine Vergütung von ca. $60 \%$ oder weniger des üblichen Lohns sittenwidrig ist. Allerdings solle es nicht weiter auf diesen Abstand ankommen und Sittenwidrigkeit zu bejahen sein, wenn der Lohn für eine Vollzeittätigkeit nicht die Existenz der Beschäftigten sichern könne. ${ }^{42}$ Auch

BAG, NZA 2009, 837; BAG, NZA 2000, 1051; BAG, AuR 2001, 510; BAG, NJW 2000, 3590; so auch schon BAG, AP Nr. 30 zu $\$ 138$ BGB.

32 BAG, DB 2004, 1432.

33 BAG, DB 2004, 1432.

34 BAG, NZA 2009, 838.

35 Aus der Rechtsprechung z.B.: ArbG Wesel, AiB 1996, 126; ArbG Hagen, NZA 1987, 610; LAG Bremen, AiB 1993, 834; LAG Berlin, NZA-RR 1998, 392; aus der Literatur z.B.: Peter, AuR 1999, 291; Reinecke (Fn. 19), S. 32; Lakies (Fn. 16), S. 341; wenn die Anwendung von $\$ 138$ BGB von subjektiven Merkmalen abgekoppelt wird: Bepler, FS für Reinhard Richardi, 2007, S. 202; a.A. Heiden, DB 2006, 2401 und Krause/Münchener Handbuch zum Arbeitsrecht, 2009, $\$ 54$ Rn. 76, die eine Orientierung am marktüblichen Entgelt verlangen.

36 Peter (Fn. 28), S. 511; Lakies (Fn. 16), S. 342.

37 Z.B.: BAG, AuR 2001, 510 (70\% der üblichen Vergütung keine Sittenwidrigkeit); BAG, 5 AZR 151/88, n.v. (74\% des Tariflohns keine Sittenwidrigkeit); BAG, AP Nr. 45 zu $\$ 242$ BGB Gleichbehandlung (69,4\% des Tariflohns keine Sittenwidrigkeit); BAG, AP Nr. 30 zu $\$ 138$ BGB (72,7 \% des Tariflohns keine Sittenwidrigkeit); BAG, 5 AZR 1008/78, n.v. (Sittenwidrigkeit bei $20 \%$ des üblichen Lohns); BAG, NZA 2006, 1354 (Sittenwidrigkeit bei Vergütung von Privatschul-Lehrkräften, die weniger als $75 \%$ der Vergütung von Lehrkräften im öffentlichen Dienst beträgt); Überblick bei: Erfurter Kommentar/Preis (Fn. 29), Rn. 3.

38 BAG, NZA 2009, 837.

39 BGH, JR 1999, 164 ff.: der BGH ist hier bei Zahlung von 66,6 \% des Tariflohns von einem auffälligen Missverhältnis zwischen Arbeitsleistung und Lohn ausgegangen.

40 Z.B. LAG Berlin-Brandenburg, Streit 2007, 168; ArbG Hamburg, AuR 2007, 445; LAG Bremen, AuR 2008, 357; LAG Hamm, AuR 2009, 134.

41 Z.B. ArbG Stralsund, AuR 2009, 182 (Sittenwidrigkeit bei $50 \%$ des Tariflohns, auch wenn im entsprechenden Tarifgebiet kaum Tariflöhne gezahlt werden. Hier wurde das Verfahren vom Sozialleistungsträger geführt, da die Arbeitnehmerin ergänzende Sozialleistungen in Anspruch nehmen musste, um ihren Lebensunterhalt zu sichern, vgl. Anmerkung Buschmann, AuR 2009, 183); ähnlicher Grenzwert: ArbG Bremen-Bremerhaven, AuR 2008, 275.

42 ArbG Bremen, AuR 2001, 232; ebenso: SG Berlin, AuR 2007, 54; SG Fulda, info also 2004, 217; a.A. BAG, NZA 2004, 971. 
müssten die Gerichte bei der Auslegung der zivilrechtlichen Generalklauseln dem internationalen Recht innerstaatliche Geltung verschaffen und Art. 4 Abs. 1 ESC beachten. ${ }^{43}$ In älteren Urteilen der Arbeitsgerichte wurde schließlich Sittenwidrigkeit bei Zahlung von 28-66\% des Tariflohns oder des üblichen Lohns bejaht ${ }^{44}$ und bei Zahlung von $70-80 \%$ des Tariflohns verneint. ${ }^{45}$ Insgesamt handelt es sich aber bei all diesen Urteilen um Einzelfallentscheidungen, die nur begrenzt verallgemeinerbar sind. ${ }^{46}$

\section{Kritik}

Die bisherige Rechtsprechung des BAG war vor allem deswegen kritikwürdig, weil sie keinen klaren Grenz- und Bezugswert für sittenwidrige Löhne definierte. ${ }^{47}$ Im Urteil vom April 2009 hat das BAG nun erstmals mit 2/3 des Tariflohns eine klare Grenze für sittenwidrige Löhne festgelegt. Dabei geht das Gericht davon aus, dass Tariflöhne eines bestimmten Wirtschaftsgebiets den objektiven Wert der Arbeitsleistung wiedergeben, wenn in diesem Wirtschaftsgebiet üblicherweise der Tariflohn gezahlt wird. Tariflöhne entsprechen dann nach Ansicht des BAG dem Marktwert der Arbeitsleistung. ${ }^{48}$ Dieser Ansatz ist grundsätzlich zu begrüßen. Das BAG bewegt sich damit im Rahmen der Tarifautonomie. Es geht davon aus, dass die Tarifautonomie die strukturelle Unterlegenheit der Beschäftigten durch kollektive Einbindung ausgleicht und ein gleichgewichtiges Aushandeln der Löhne ermöglicht. ${ }^{49}$

Soll der Tariflohn aber auch dann noch den Wert der Arbeitsleistung bestimmen, wenn er einen nicht existenzsichernden Niedriglohn festlegt? So würde der objektive Wert der Arbeitsleistung z.B. einer/s Friseurin/s in Sachsen beim Tariflohn von 3,06 €/Stunde liegen, Sittenwidrigkeit wäre bei Unterschreitung dieses Lohns um $1 / 3$ (also erst bei 2,04 €/Stunde) gegeben. ${ }^{50}$ Niedrige Tariflöhne können somit noch niedrigere Löhne rechtfertigen. Sie werden nicht nur von sog. „gelben Gewerkschaften“vereinbart, deren Gewerkschaftseigenschaft zu Recht bezweifelt wird, ${ }^{51}$ sondern finden sich auch in Tarifverträgen etablierter Gewerkschaften. ${ }^{52}$ Die Orientierung des BAG mit dem Tariflohn am Marktwert der Arbeitsleistung führt bei Marktversagen zu absurden Lohnwuchergrenzen. ${ }^{53}$

43 ArbG Bremen, AuR 2001, 233; ebenso: SG Berlin, AuR 2007, 55: wesentliches Kriterium für die Gestaltung von Arbeitslöhnen sei neben Art. 1, Art. 2 Abs. 1, Art. 20 Abs. 1 GG auch Art. 4 ESC.

44 Z.B. ArbG Ludwigshafen, 3 Ca 278/96, n.v. (zitiert nach Tschöpe, DB 2002, 1831: 64 \% des Marktlohns); ArbG Reutlingen, AiB 1996, 499 (57,2 \% des Tariflohns); ArbG Wesel, AiB 1996, 126 (43\% des Tariflohns); LAG Bremen, AiB 1993, 834 (34 \% - $28 \%$ des Vergleichslohns); LAG Düsseldorf, DB 1978, 165 (60\% oder weniger des üblichen Lohns oder Tariflohns).

45 ArbG Hagen, NZA 1987, 610 (70 \% des niedrigsten Lohns eines vergleichbaren Tarifvertrags); LAG Köln, BB 1986, 2057.

46 Auch in der Literatur gibt es verschiedene Ansätze zur Bestimmung des Grenzwertes: Erfurter Kommentar/Preis (Fn. 29), Rn. 3 (Hälfte des Tariflohns); Hanau/Münchener Handbuch zum Arbeitsrecht, 2000, 563 Rn. 6 (Hälfte des Marktlohns); Lakies (Fn. 16), S. 342; Peter (Fn. 35), S. 293; Reinecke (Fn. 19), S. 32 (66,7 \% des Tariflohns); Krause (Fn. 35), Rn. 80 (66,7 \% des üblichen Lohns).

47 Ebenso: Bayreuther (Fn. 9), S. 2022.

48 BAG, NZA 2009, 839.

49 Vgl. BVerfGE 84, 212 (229).

50 Vgl. Böckler-Box „Lohngrenzen Relative Sittlichkeit“: http://www.boeckler-boxen.de/5684.htm (abgerufen am 12.2.2010).

51 Zweifel z.B. an der Tariffähigkeit der Tarifgemeinschaft Christlicher Gewerkschaften für Zeitarbeit und PSA (CGZP) äußern: Ulber, AuR 2008, 297 f.; Schüren, NZA 2008, 453 f.; Deinert, NZA 2009, 1178; ArbG Berlin, AuR 2009, 276; Auch die Tariffähigkeit der Gewerkschaft der privaten Brief- und Zeitungszustellern (GNBZ) wird bezweifelt: ArbG Köln, AuR 2009, 100; ArbG Berlin, AuR 2009, 178; LAG Köln, AuR 2009, 316.

52 Vgl. WSI-Tarifarchiv: http://www.boeckler.de/show_mindestlohn.html (abgerufen am 12.2.2010).

53 Däubler, Das Arbeitsrecht 2, 2009, Rn. 816; Bayreuther (Fn. 9), S. 2025. 
Nach vorliegendem Verständnis hingegen gibt es - wie auch das Sozialgericht

Berlin meint - einen durch die Verfassung vorgegebenen Mindestwert der Arbeitsleistung, der bei Vollzeittätigkeit einer sozialstaatlich-menschenwürdigen Existenzsicherung entspricht. Es gibt elementare Gerechtigkeitsanforderungen an die Lohngestaltung, die sich aus Art. 20 Abs.1, Art. 1 Abs. 1 und Art. 2 Abs. 1 GG ableiten lassen. Diese Grenze liegt der gesamten Rechtsordnung zugrunde und bindet auch den Arbeitsmarkt. ${ }^{54}$ Wenn Tariflöhne diese Grenze unterschreiten, dürfen sie nicht als Maßstab herangezogen werden, denn auch Art. 9 Abs. 3 GG schützt keine tarifvertragliche Regelung, die diesen verfassungsrechtlichen Rahmen verlässt. ${ }^{55}$ Die mit der Koalitionsfreiheit verbundene Hoffnung auf ein gleichgewichtiges Aushandeln der Löhne erfüllt nicht ihren Zweck, wenn Tariflöhne nicht existenzsichernd sind. Dann versagt das Schutzkonzept der Tarifautonomie. ${ }^{56}$

Aus Art. 20 Abs. 1 GG folgt, dass der Staat die Pflicht hat, die Mindestvoraussetzungen für ein menschenwürdiges Dasein zu schaffen. ${ }^{57}$ Aus Art. 20 Abs. 1 und Art. 1 Abs. 1 GG erwächst auch ein individueller Anspruch auf ein menschenwürdiges Dasein und das soziokulturelle Existenzminimum. ${ }^{58}$ Dies bildet die Basis für die freie Entfaltung der Persönlichkeit. ${ }^{59}$ Zudem umfasst nach der Rechtsprechung des Bundesverfassungsgerichts Art. 12 GG nicht nur die Freiheit, einen Beruf auszuüben, sondern auch, eine angemessene Vergütung zu fordern. ${ }^{60}$ Im Niedriglohnbereich erzielen Beschäftigte aber trotz Vollzeittätigkeit kein existenzsicherndes Einkommen. Daher sind sie vom gesellschaftlichen Wohlstand ausgeschlossen und an der Entfaltung ihrer Persönlichkeit gehindert. Niedriglohnarbeitsverhältnisse verstoßen gegen Art. 20 Abs. 1, Art. 1 Abs. 1 und Art. 12 Abs. 1 GG. ${ }^{61}$ Bei grundrechtsrelevant gestörter Vertragsparität müssen neben dem Staat ${ }^{62}$ auch die Gerichte schützend eingreifen: Sie sind zur Inhaltskontrolle am Maßstab der Generalklauseln verpflichtet, ${ }^{63}$ die sie so auslegen müssen, dass die Grundrechte im Privatrecht zur Geltung kommen. ${ }^{64}$

\section{Die ESC als absolute Lohnuntergrenze zur Bestimmung von \138BGB}

Um den Grundrechten bei der Auslegung von $\$ 138$ BGB mehr Geltung zu verschaffen und mit dem Lohn auch das soziokulturelle Existenzminimum der Beschäftigten zu sichern, sollten absolute Lohnuntergrenzen zur Bestimmung der Sittenwidrigkeit herangezogen werden, die den Wert der Arbeitsleistung danach bestimmen, was notwendig ist für ein existenzsicherndes Leben. In Literatur und

SG Berlin, AuR 2007, 55.

SG Berlin, AuR 2007, 54 f.; zustimmend Peter, AuR 2007, 57.

Vgl. Peter (Fn. 14), S. 70.

BVerfGE 82, 60 (85); BVerfGE 87, 153 (169ff.); BVerfGE 99, 246 (259ff.); BVerfGE 107, 27 (48); BVerfGE 112, 268 (281); BVerfG, NJW 2008, $1871 \mathrm{f}$.

BVerfG, NJW 2010, 505 (Rn. 133 ff.) („Hartz IV-Urteil“); BVerfGE 40, 121 (133); BVerfGE 82, 60 (85); Hessisches Landessozialgericht, AuR 2008, 452; SG Berlin, AuR 2007, 55; Jarass/Pieroth, GG für die Bundesrepublik Deutschland, 2007, Art. 20, Rn. 142; Brall, Zum Rechtsbegriff des Sozialen, 2006, S. 33; Soria, JZ 2005, 645; a.A. Gröschner/Dreier, Grundgesetzkommentar, Band II, 2006, Art. 20 GG (Sozialstaat) Rn. 34 .

Nußberger, DVBl. 2008, 1085; vgl. auch Zachert (Fn. 20), S. 107 (mit Verweis auf Sinzheimer, der von einer dialektischen Wechselbeziehung sozialer Gerechtigkeit und menschlicher Freiheit ausgegangen sei, wodurch die eine in der anderen aufgehoben sei.).

BVerfGE 54, 251 (271); BVerfGE 83, 1 (13); BVerfGE 88, 145 (159).

So auch: Däubler/Lakies (Fn. 8), Rn. 24a.

Vgl. zur sog. Schutzpflicht des Staates, die das BVerfG aus Art. 20 Abs. 1, Art. 2 Abs. 1 und Art. 12 GG ableitet: BVerfGE 81 (242); BVerfGE 89 (214); BVerfGE 97 (169).

Vgl. BVerfGE 81, 242 (254f.); BVerfGE 89, 214 (232 f.); BVerfG, NJW 1994, 2750; BVerfG, ZIP 1996, 957.

So auch: ArbG Bremen, AuR 2001, 233. 
Rechtsprechung werden verschiedene absolute Lohnuntergrenzen diskutiert. ${ }^{65}$ Als solche Lohnuntergrenze kommt auch Art. 4 Abs. 1 ESC in Betracht, der das Recht der Beschäftigten auf ein gerechtes Arbeitsentgelt festschreibt, das ausreicht, um sich und ihren Familien einen angemessenen Lebensstandard zu sichern.

\subsection{Hintergrund}

Die ESC ist 1961 als völkerrechtlicher Vertrag und 1996 als Revidierte Sozialcharta im Rahmen des Europarats ${ }^{66}$ zustande gekommen. Sie schreibt - als Komplementärvertrag zur Europäischen Menschenrechtskonvention (EMRK) mit den politischen und bürgerlichen Rechten ${ }^{67}$ - einen Katalog sozialer Rechte fest. ${ }^{68}$ Die Revidierte Sozialcharta trat 1999 in Kraft und wurde von 24 Staaten ratifiziert (Stand: Mai 2008). ${ }^{69}$ Sie umfasst die Zusatzprotokolle der alten Sozialcharta und ergänzt den Bestand um weitere soziale Rechte. ${ }^{70}$ Die Bundesrepublik Deutschland hat der revidierten Sozialcharta bislang noch nicht zugestimmt. ${ }^{71}$ Allerdings hat sie 1964 nach Art. 59 Abs. 2 GG die alte Sozialcharta ratifiziert. ${ }^{72}$ Sie hat auch Art. 4 Abs. 1 ESC anerkannt und sich so international zum Schutz vor Niedriglöhnen verpflichtet. ${ }^{73}$

\subsection{Das Europäische Komitee der sozialen Rechte (EKSR)}

Da die ESC im Gegensatz zur EMRK nicht gerichtlich durchgesetzt werden kann, kann nur das sog. Europäische Komitee der sozialen Rechte (EKSR) ${ }^{74}$ auf Grundlage von Berichten der Mitgliedstaaten überprüfen, ob die Staaten ihren Verpflichtungen aus der ESC nachkommen. ${ }^{75}$ Das EKSR wird vom Ministerko-

65 So z.B. die Leistungen der Grundsicherung nach SGB II, die Pfändungsfreigrenzen nach $₫ 850 c$ ZPO und das steuerfreie Existenzminimum nach $\$ \$$ 32a Abs. 1 Nr. 1 EStG, dazu: Krause (Fn. 35), Rn. 81 ff.; Däubler/Lakies (Fn. 9), Rn. 47 ff. (mwN). Die Grundsicherung im SGB II als Lohnwuchergrenze ablehnend: BAG, NZA 2004, 972; Franke, Lohnwucher, 2003, S. 122; Henssler/Sittard, RdA 2007, 161; Krause (Fn. 35), Rn. 81 ff. - bejahend: SG Berlin, AuR 2007, 54 f.; SG Fulda, info also 2004, 217; ArbG Bremen, AuR 2001, 231; Lakies (Fn. 16), S. 342; Peter (Fn. 14), S. 210 f.; $\$ 850$ c ZPO als Lohnwuchergrenze ablehnend: BAG, NZA 2004, 972 - bejahend: ArbG Bremen, AuR 2001, 233; \32a Abs. 1 Nr. 1 EStG als Lohnwuchergrenze ablehnend: Krause (Fn. 35), Rn. 82 - bejahend: ArbG Bremen, AuR $2001,233$.

66 Der Europarat wurde am 5. Mai 1949 auf Anregung von W. Churchill gegründet, um den Wiederaufbau in Europa auf Grundlage gemeinsamer Werte zu stärken. Er ist die älteste zwischenstaatliche politische Organisation Europas und von supranationalen Organisationen wie der EU zu trennen. Weitere wichtige vom Europarat verabschiedete Konventionen sind die Europäische Konvention zum Schutz der Menschenrechte und Grundfreiheiten (EMRK) und die Antifolter-Konvention - vgl. dazu Hailbronner/Vitzthum, Völkerrecht, 2007, Rn. 236 ff.; Krebber, JZ 2008, 57; Däubler, AuR 1998, 144.

67 Konvention zum Schutze der Menschenrechte und Grundfreiheiten vom 4.11.1950: BGBl. II 1952, S. 686, 953; dazu eingehend: Hailbronner/Vitzthum (Fn. 66), Rn. 238 ff.

68 Neubeck, Die Europäische Sozialcharta und deren Protokolle, 2001, S. 56 (mwN); Lörcher, Das Recht auf angemessenes Arbeitsentgelt nach der Europäischen Sozialcharta, in: Sterkel (Hrsg.), Mindestlöhne gegen Lohndumping, 2006, S. 217; Dötsch, AuA 2001, 28.

69 Stein/von Butlar, Völkerrecht, 2009, Rn. 1054.

70 Zur Revidierten Sozialcharta eingehend: Neubeck (Fn. 69), S. 78 ff.; Hailbronner/Vitzthum (Fn. 67), Rn. $255 \mathrm{ff}$.

71 Stein/von Butlar (Fn. 69), Rn. 1054; Dötsch (Fn. 68), S. 27.

72 BGBl. II 1964, S. $1261 \mathrm{f}$.

73 Hailbronner/Vitzthum(Fn. 66), Rn. 256; Däubler/Lakies (Fn. 8), Rn. 21; Lörcher (Fn. 68), S. 217 f.; nach Neubeck (Fn. 68), S. 139 ff. sind die von Deutschland nicht ratifizierten Artikel: Art. 4 Abs. 4, Art. 7 Abs. 1, Art. 8 Abs. 2, Art. 8 Abs. 4, Art. 10 Abs. 4.

74 Früher auch „Sachverständigenausschuss“ oder „Ausschuss unabhängiger Sachverständiger“ genannt.

75 Lörcher (Fn. 68), S. 218; Neubeck (Fn. 68), S. 119 ff.; vgl. zum Staatenberichtsverfahren als Instrument internationaler Rechtsdurchsetzung beim UN-Pakt für bürgerliche und politische Rechte: Liese, Die Friedens-Warte 2006, 51. 
mitee ${ }^{76}$ gewählt. ${ }^{77}$ Aufgrund seiner Prüfungen formuliert das EKSR seine Beratungsergebnisse in den sog. Schlussfolgerungen, die dann der Regierungsausschuss erhält und nach politischen, sozialen und wirtschaftlichen Kriterien entscheidet, ob bei Verstößen dem Ministerkomitee Abhilfemaßnahmen für den betroffenen Vertragsstaat vorgeschlagen werden. Das Ministerkomitee kann im äußersten Fall mit einer 2/3-Mehrheit diese Abhilfemaßnahmen als sog. Empfehlungen beschließen, die die Vertragsstaaten dazu anhalten sollen, ihr nationales Recht in Einklang mit der ESC zu bringen; eine Pflicht dazu besteht nach der ESC aber nicht. ${ }^{78}$

Nach den Schlussfolgerungen V des EKSR zu Art. 4 Abs. 1 der ESC gelten derzeit $60 \%$ des nationalen Netto-Durchschnittslohns als Maßstab für einen angemessenen Lohn. Die Vertragsstaaten können aber bis zu einer Grenze von 50 \% des nationalen Netto-Durchschnittslohns nachweisen, dass dennoch ein angemessener Lebensstandard vorliegt. ${ }^{79}$ Der nationale Netto-Durchschnittslohn in Deutschland betrug $200712,98 €$ pro Stunde, also 2167,00 € netto pro Monat. ${ }^{80}$ $60 \%$ davon machen $1300,00 €$ als monatlichen Nettolohn aus. Ein Unterschreiten dieser Grenze könnte im Rahmen der Auslegung von $\$ 138$ BGB durch die Gerichte eine Orientierung für die Sittenwidrigkeit von Löhnen bilden.

\subsection{Innerstaatliche Geltung der ESC}

$\mathrm{Zu}$ klären ist zunächst, inwieweit die ESC innerstaatliche Geltung beansprucht und von deutschen Gerichten beachtet werden muss. Das Grundgesetz weist dem Völkerrecht eine wichtige Rolle zu. Dies zeigt sich am Grundsatz der sog. „Völkerrechtsfreundlichkeit“, der u.a. in den Art. 1 Abs. 2, Art. 24 bis 26, Art. 59 und Art. 100 Abs. 2 GG und der Präambel des Grundgesetzes zum Ausdruck kommt. ${ }^{81}$ Daraus ergibt sich für Regierung, Verwaltung und Gerichte das Gebot, sowohl einfache Gesetze als auch die Verfassung im Einklang mit den für die Bundesrepublik verbindlichen Völkerrechtsnormen auszulegen. ${ }^{82}$ Innerstaatlich verbindlich sind Völkerrechtsnormen, wenn sie - wie die Ausgangsfassung der ESC - von Deutschland gem. Art. 59 Abs. 2 GG ratifiziert worden sind. Durch die Ratifizierung werden völkerrechtliche Verträge in deutsches Recht transformiert und stehen im Rang eines Bundesgesetzes. Entsprechend müssen sie von deutschen Gerichten im Rahmen methodisch vertretbarer Auslegung innerstaatlichen Rechts angewandt werden. ${ }^{83}$ Insbesondere müssen die Gerichte nach Ansicht des Bundesverfassungsgerichts bei mehreren möglichen Interpretationen deutscher Rechtsnormen diejenige wählen, die den Anforderungen des Völkermit jeweils einer Stimme vertreten, soweit sie die ESC ratifiziert haben - vgl. Däubler (Fn. 67), S. 144.

77 Kothe/Doll, ZESAR 2003, 394; Lörcher (Fn. 68), S. 218.

78 Ausführlich zum Kontrollverfahren: Neubeck (Fn.68), S. 86 f.; Hailbronner/Vitzthum (Fn. 66), Rn. 257; Kothe/Doll (Fn. 77), S. 394; Däubler (Fn. 66), S. 14; In der Literatur werden die Kontrollmöglichkeiten z.T. als unbefriedigend kritisiert, da auch bei Feststellung einer Verletzung der ESC durch das EKSR die Einflussmöglichkeiten auf die Staaten begrenzt seien: vgl. Soria (Fn. 58), S. 646; Dötsch (Fn. 68), S. 28.

79 Däubler/Lakies (Fn. 8), Rn. 22a, Krause (Fn. 35), Rn. 84.

80 Grundlage für die Berechnung ist eine alleinstehende Person, geb. nach 1944, keine Kinder, gesetzliche Krankenkasse 14,9\%, Kirchensteuer, Bundesland Berlin, Steuerklasse I, Jahr 2009, 38,5 Stunden-Woche (=167 Stunden/Monat), vgl. Bispinck/Schulten (Fn. 10), S. 156.

81 BVerfGE 111, 307 (318); BVerfGE 63, 343 (370); BVerfGE 31, 58 (75f.); vgl. auch Sommermann/ v. Mangoldt u.a., GG II, 2005, Art. 20, Rn.254; Lörcher, AuR 1991, 104; Stein/von Butlar (Fn. 69), Rn. 192; zur Entwicklung des Grundsatzes der Völkerrechtsfreundlichkeit des GG: Neubeck (Fn. 68), S. $183 \mathrm{f}$.

82 BVerfG, NJW 2007, 499 (Rn. 54/58); BVerfGE 111, 307 (318); vgl. auch Stein/von Butlar (Fn. 69), Rn. 192; Sommermann/v. Mangoldt (Fn. 81), Rn. 254.

83 Däubler/Lakies (Fn. 8), Rn. 22a; Lorenzmeier, HFR 13/2008, 137 f.; Krause (Fn. 35), Rn. 85. 
rechts am besten Rechnung trägt. ${ }^{84}$ Ebenso werden durch die Ratifizierung alle Staatsorgane mittelbar zur Durchsetzung des Völkerrechts verpflichtet. ${ }^{85}$

Diese Prinzipien hat das Bundesverfassungsgericht am Beispiel der EMRK entwickelt: Es hat die EMRK und die Rechtsprechung des Europäischen Gerichtshofs für Menschenrechte (EGMR) zur Konkretisierung des Grundgesetzes herangezogen und dabei festgestellt, dass sie Bestandteil des positiven Rechts im Range eines Bundesgesetzes und von den Gerichten bei der Auslegung innerstaatlichen Rechts zu beachten sind. ${ }^{86}$ Diese Rechtsprechung ist nach vorliegender Auffassung auf die ESC übertragbar: Da die ESC wie auch die EMRK ein im Rahmen des Europarates zustande gekommener völkerrechtlicher Vertrag ist, subjektive Grund- und Menschenrechte auf europäischer Ebene definiert und von der Bundesrepublik ratifiziert worden ist (zumindest Art. 4 Abs. 1 ESC), ist die ESC wie auch die EMRK bei der Auslegung des innerstaatlichen Rechts heranzuziehen. ${ }^{87} \mathrm{Ob}$ für die ESC dieselben Grundsätze gelten sollen wie für die EMRK, hat das Bundesverfassungsgericht aber bisher offen gelassen. Dabei hat es allerdings auch die Alternative ausgeschlossen, die ESC könnte gänzlich „wirkungslos" sein. ${ }^{88}$

Das BAG hat 1984 in einem Grundsatzurteil zum Warnstreik entschieden, die Gerichte müssten gesetzliche Lücken anhand von Wertentscheidungen des Gesetzgebers ausfüllen und hätten dabei auch völkerrechtliche Verpflichtungen zu beachten. Die Bundesrepublik Deutschland dürfe dem Streikrecht nur solche Grenzen setzen, die nach Art. 31 Abs. 1 ESC zulässig seien. Die ESC bilde eine wichtige Grundlage für die Auslegung und Fortbildung des Arbeitsrechts durch die Arbeitsrechtsprechung. ${ }^{89}$ Im Urteil vom 10.12.2002 mahnt das BAG schließlich eine Überprüfung des nationalen Streikrechts im Hinblick auf Art. 6 ESC an und setzt sich dabei auch mit der Auffassung des EKSR über die Auslegung von Art. 6 ESC auseinander. ${ }^{90}$ In einer Entscheidung zum Lohnwucher von 2004 hat sich das BAG jedoch mit der Feststellung begnügt, die ESC räume einzelnen Personen keine einklagbaren Ansprüche ein. ${ }^{91}$ Es hat sich aber nicht weiter mit der innerstaatlichen Geltung und der Frage befasst, inwiefern $\ 138$ BGB völkerrechtskonform auszulegen sein könnte. ${ }^{92}$

Von der innerstaatlichen unmittelbaren Geltung zu trennen ist hingegen die Frage, ob die Bestimmungen der ESC innerstaatlich unmittelbar anwendbar sind. ${ }^{93}$ Dies ist der Fall, wenn aus ihnen unmittelbare Rechtsfolgen für den Ein-

84 BVerfGE 58, 1 (34); BVerfGE 59, 63 (89); BVerfGE 111, 307 (317).

85 BVerfG, NJW 2007, 499, Rn. 58; BVerfGE 111, 307 (318); BVerfGE 112, 1 (26); vgl. auch Lorenzmeier (Fn. 83), S. 139; Kempen/v. Mangoldt u.a., GG II, 2005, Art. 59 Abs. 2 GG, Rn. 95; Böhmert, Das Recht der ILO und sein Einfluss auf das deutsche Arbeitsrecht im Zeichen der europäischen Integration, 2002, S. 154.

86 BVerfGE 74, 358 (370); BVerfGE 82, 106 (120); BVerfGE 111, 307 (316f.).

87 Sommermann/v. Mangoldt (Fn. 81), Rn. 136; Lörcher (Fn. 68), S. 219; jedoch weist Däubler (Fn. 66), S. 147 f. darauf hin, dass in Art. 53 EMRK eine ausdrückliche Verpflichtung der Vertragsstaaten festgelegt sei, Entscheidungen des EGMR zu befolgen. Eine solche Verpflichtung sei in der ESC nicht zu finden.

88 BVerfGE 58, 233 (254).

89 BAG, AP Nr. 81 zu Art. 9 GG - Arbeitskampf; vgl. auch: Dötsch (Fn. 68), S. 28; Konzen, JZ 1986, 158; Kothe/Doll (Fn. 77), S. 393.

90 BAG, NZA 2003, 734; interessant sind in diesem Kontext auch die Urteile des EGMR vom 12.11.2008 - 34503/07 (Demir und Baykara/Türkei) und vom 21.4.2009 - 68959/01 (Enerji Yapi-Yol sen/Türkei), in denen das EGMR die Annahme eines Streikrechts für Beamte u.a. auf die ESC und deren Auslegung durch das EKSR stützt - in der Literatur wird daher eine Überprüfung des Streikverbots im deutschen Beamtenrecht gefordert: vgl. Seifert, KritV 2008, 357; Lörcher, AuR 2009, 229.

91 BAG, NZA 2004, 974.

92 Vgl. Däubler (Fn. 53), Rn. 816b.

93 Zur uneinheitlichen Verwendung des Begriffs „,unmittelbare Anwendbarkeit“ in der Literatur: Neubeck (Fn. 68), S. 162, Fn. 60. 
zelfall abgeleitet werden können (sog. „self-execting“-Norm). Dafür ist der Norminhalt entscheidend. ${ }^{94}$ Diese Frage ist stark umstritten. ${ }^{95}$ In Bezug auf Art. 4 ESC wird überwiegend angenommen, die Norm sei für den Einzelfall nicht unmittelbar anwendbar, da Art. 4 ESC seinem Wortlaut nach nur als Verpflichtung der Vertragsstaaten ausgestaltet sei, ein entsprechendes Recht anzuerkennen. ${ }^{96}$ Andererseits wird auch zunehmend die Position vertreten, dass soziale Rechte insgesamt - also auch die in der ESC geregelten Rechte - justiziabel seien. ${ }^{97}$ Diese Ansicht hat mit der Verabschiedung des neuen Fakultativprotokolls zum UN-Pakt für wirtschaftliche, soziale und kulturelle Rechte (UN-Sozialpakt) am 10.12.2008 durch die UN-Generalversammlung neue Rückendeckung bekommen: ${ }^{98}$ Einzelpersonen können nun den eigenen Staat wegen Verletzung ihrer sozialen Rechte nach dem UN-Sozialpakt vor dem entsprechenden UNAusschuss zur Rechenschaft ziehen, wenn der Staat das Protokoll ratifiziert hat. Die Einzelheiten dieses Streits sollen hier jedoch nicht weiter ausgeführt werden. Denn für die innerstaatliche Geltung der ESC ist deren unmittelbare Anwendbarkeit keine Voraussetzung, weil sich das Zustimmungsgesetz nach Art. 59 Abs. 2 GG auf den ganzen völkerrechtlichen Vertrag und nicht nur auf dessen unmittelbare Bestimmungen bezieht. ${ }^{99}$

Somit kann festgehalten werden, dass Art. 4 Abs. 1 ESC zumindest innerstaatlich unmittelbar geltendes Recht darstellt und daher von den deutschen Gerichten im Rahmen methodisch vertretbarer Auslegung von $\$ 138$ BGB angewandt werden muss. ${ }^{100}$

\subsection{Die Beratungsergebnisse des EKSR}

Daran anknüpfend stellt sich die Frage, inwieweit die durch das EKSR bestimmte Grenze für einen angemessenen Lohn nach Art. 4 Abs. 1 ESC (60\% des nationalen Netto-Durchschnittslohns) bei der Auslegung von $\$ 138$ BGB zu berücksichtigen ist.

In der ESC selbst findet sich keine Regelung dazu. Daher geht ein Teil der Literatur davon aus, dass eine unmittelbare formell-rechtliche Bindung der nationalen Gerichte an die Auffassung des EKSR nicht bestehe. Denn hätte die ESC dies gewollt, hätte es im Vertragstext dazu eine ausdrückliche Regelung gegeben. ${ }^{101}$ Eine direkte Einwirkung der ESC auf das innerstaatliche Recht sei auch historisch nicht gewollt. ${ }^{102}$ Andere hingegen vertreten die Ansicht, dass den Beratungsergebnissen eine besondere Autorität zukomme, auch wenn sie formal nicht bin-

Koenig/v. Mangoldt u.a., GG II, 2005, Art. 25 Rn. 45; Kunig/Vitzthum, Völkerrecht, 2007, Rn. 41. Vgl. Lorenzmeier (Fn. 83), S. 138 (mwN); Konzen (Fn. 89), S. 162; Soria (Fn. 58), S. 646; Neubeck (Fn. 68), S. 166 ff.; Däubler, Das Arbeitsrecht 1, 2006, Rn. 20.

BAG, NZA 2004, 974; ebenso: Krause (Fn. 35), Rn. 31; Däubler/Lakies (Fn. 8), Rn. 22a.

Fischer-Lescano, ZERP-Diskussionspapier 2/2010, S. 22 (i.E.), der parallel zur EMRK eine gerichtsförmige Ausgestaltung der ESC mit Individual- und Kollektivbeschwerden fordert; vgl. Wimalasena, KJ 2008, 2 ff., der meint, eine kategorische Ablehnung der Justiziabilität sozialer Rechte sei nicht überzeugend zu vertreten; weitere Nachweise bei Schneider, Die Justiziabilität wirtschaftlicher, sozialer und kultureller Menschenrechte, 2004, S. $9 \mathrm{ff}$.

UN-Dok. A/RES/63/117, Anhang v. 10.12.2008: http://www2.ohchr.org/english/law/docs/A.RES. 63.117_en.pdf (abgerufen am 1.3.2010); dazu eingehend: Aichele, Vereinte Nationen 2009, 72, der meint, dass das Protokoll die vorgebrachten Zweifel an der Einklagbarkeit der im UN-Sozialpakt niedergelegten wirtschaftlichen, sozialen und kulturellen Rechte entkräfte.

So auch: Lorenzmeier (Fn. 83), S. 138; Kempen/v. Mangoldt (Fn. 85), Rn. 95; Kunig/Vitzthum (Fn. 94), Rn. 111.

Ebenso: ArbG Bremen, AuR 2001, 231; SG Berlin, AuR 2007, 55; SG Düsseldorf, S. 31 AS 317/07 (n.V.); vgl. auch Zachert, Anm. zu BAG, AP Nr. 63 zu $\$ 138$ BGB; Hanau (Fn. 46), Rn. 3; Däubler (Fn. 66), S. 148.

Voßberg, Inhaltskontrolle von arbeitsvertraglichen Entgelthöheregelungen, 2005, S. 175 ff. (mwN). Neubeck (Fn. 68), S. 193 f. 
dend seien. Dies werde durch die gerichtsähnliche Ausgestaltung und Publizität der Verfahren und Schlussfolgerungen deutlich. ${ }^{103}$ Das EKSR zeichne sich zudem durch einen hohen Grad an Unabhängigkeit und Sachkunde ihrer Mitglieder aus. Ihre Beratungsergebnisse stellten eine einheitliche Auslegung der ESC in allen Vertragsstaaten sicher. ${ }^{104}$ Mit Unterzeichnung der ESC durch die Bundesrepublik sei auch die Arbeit des EKSR implizit anerkannt worden. ${ }^{105}$ Die nationalen Gerichte hätten sich mit ihren Aussagen zumindest auseinanderzusetzen. ${ }^{106}$ Einige Autoren beziehen sich auf einen Vergleich mit den von der ILO gesetzten sozialen Standards, die auch ohne formelle Bindung einen gewissen Richtliniencharakter hätten. ${ }^{107}$ Andere verweisen darauf, dass das Bundesverfassungsgericht die Konkretisierung des Grundgesetzes durch die Urteile des EGMR und deren Beachtung bei der Auslegung innerstaatlichen Rechts durch die nationalen Gerichte verlange, obwohl auch diesen Urteilen innerstaatlich keine formelle Bindungswirkung zukomme. ${ }^{108}$

Auch nach hier vertretener Ansicht muss der Maßstab des EKSR als ausschlaggebende Konkretisierung der ESC-Normen betrachtet und durch die Gerichte bei der Auslegung von $\$ 138$ BGB berücksichtigt werden. ${ }^{109}$ Die Zusammensetzung und Aufgaben des EKSR deuten auf eine besondere Autorität und Unabhängigkeit seiner Beratungsergebnisse hin, die eine einheitliche Anwendung der ESC sicherstellen. Es würde dem Grundsatz der Völkerrechtsfreundlichkeit widersprechen, wenn diese rechtlich irrelevant wären. ${ }^{110}$ Der Auslegung des EKSR sollte nur im Einzelfall bei offensichtlicher Unrichtigkeit nicht gefolgt werden. ${ }^{111}$

\section{Ergebnis}

Die ESC ist für die Bundesrepublik völkerrechtlich verbindlich und stellt damit innerstaatlich unmittelbar geltendes Recht dar. Wegen der Völkerrechtsfreundlichkeit des Grundgesetzes müssen deutsche Gerichte auch einfache Gesetze im Einklang mit der ESC auslegen. Dabei ist der Maßstab des EKSR als ausschlaggebende Konkretisierung der ESC zu betrachten. Daher müssen die Gerichte die Grenze des EKSR für einen angemessenen Lohn nach Art. 4 Abs. 1 ESC bei der Auslegung von $\$ 138$ BGB beachten. Dabei kann jedoch nicht jedes Unterschreiten dieser Grenze Sittenwidrigkeit indizieren, da es bei der ESC um eine Angemessenheitsgrenze geht. Die Angemessenheitsgrenze gewährleistet aber eine Orientierung, deren Abweichen nach unten Sittenwidrigkeit signalisiert, wobei die Größe des Abstands zwischen Angemessenheit und Sittenwidrigkeit bestimmt werden muss. In der Literatur wird zu Recht vorgeschlagen, bei $60 \%$ des nationalen Brutto-Durchschnittslohns (entspricht ca. $50 \%$ des nationalen Netto-Durchschnittslohns) von Sittenwidrigkeit auszugehen. ${ }^{112}$ Damit gelangt man zu einer Sittenwidrigkeitsgrenze in Höhe von ca. 1083,83 € Nettolohn/Monat für eine Vollzeittätigkeit.

Kothe/Doll (Fn. 77), S. 395 f.

Däubler (Fn. 66), S. 148; Neubeck (Fn. 68), S. 201; Feldhoff, Anm. zu SG Düsseldorf, S 31 AS 317/07, jurisPR-ArbR 25/2009, Anm. 3, C.

Peter (Fn. 14), S. $127 \mathrm{f}$.

Däubler (Fn. 53), Rn. 816b; Däubler (Fn. 95), Rn. 20.

Kothe/Doll (Fn. 77), S. 395; zur Spruchpraxis der Kontrollgremien der ILO: Lörcher (Fn. 81), S. 103 f. Neubeck (Fn. 68), S. 194 (mwN).

Peter (Fn. 14), S. 120 ff.; Däubler/Lakies (Fn. 8), Rn. 22a; Feldhoff (Fn. 104).

Däubler (Fn. 66), S. 148.

Zur vergleichbaren Diskussion beim Menschenrechtsausschuss des Internationalen Pakts über wirtschaftliche, soziale und kulturelle Rechte: Lorenzmeier (Fn. 83), S. 131 ff.

Peter (Fn. 14), S. 122. 
Eine gesetzliche Festschreibung der „Rechtsprechung zum Verbot sittenwidriger Löhne" mit der Lohnwuchergrenze von $2 / 3$ des Tariflohns ist problematisch, wenn der Tariflohn selbst einen Niedriglohn darstellt. Mit niedrigen Tariflöhnen als Vergleichsmaßstab bei $\ 138$ BGB kann Lohndumping nicht verhindert werden. Stattdessen könnte versucht werden, die Lohnwuchergrenze an der Spruchpraxis des EKSR zu Art. 4 Abs. 1 ESC zu orientieren. Allerdings ist nicht zu erwarten, dass der Gesetzgeber in nächster Zeit in dieser Hinsicht aktiv werden wird, sowenig auch mit der Einführung eines gesetzlichen Mindestlohns zu rechnen ist. Daher sollten die Gerichte versuchen, ihrer Verpflichtung zur völkerrechtskonformen Auslegung nationalen Rechts nachzukommen und Art. 4 Abs. 1 ESC bei der Auslegung von $\ 138$ BGB zu berücksichtigen. Damit wäre sichergestellt, dass die deutsche Rechtsordnung nicht gegen die ESC verstößt. Ein solches Vorgehen ist zudem wegen Art. 20 Abs. 1 und Art. 1 Abs. 1 GG geboten. So könnte die richterliche Entgeltkontrolle Beschäftigten einen Schutz vor Niedriglöhnen bieten. Allerdings setzt eine richterliche Entgeltkontrolle voraus, dass Beschäftigte gegen ihre Lohnvereinbarungen klagen. ${ }^{113}$ Dies kommt aufgrund der sozio-ökonomischen Abhängigkeit der Niedriglohnbeschäftigten von ihren ArbeitgeberInnen aber nur selten vor. ${ }^{114}$ Zudem ist es auch eigentlich Aufgabe der Gesetzgebung und nicht der Rechtsprechung, strukturelle Probleme zu lösen. ${ }^{115}$ So bleibt die Forderung nach einem einheitlichen gesetzlichen Mindestlohn weiterhin aktuell. Nur der gesetzliche Mindestlohn kann einen lückenlosen Schutz gegen Lohndumping gewährleisten. Dabei sollte er so hoch sein, dass durch Vollzeittätigkeit wieder eine sozialstaatlich-menschenwürdige Existenzsicherung von Beschäftigten und ihren Familien möglich wird. gerichtlichen Rechtsschutz in Anspruch nehmen.

114 Vgl. Bieback, RdA 2000, 208 f.; in der Literatur werden auch weitere Mängel der richterlichen Entgeltkontrolle diskutiert: Mindeststandards können nur für Einzelfälle durchgesetzt werden. Da die Betroffenen sich erst auf den sittenwidrigen Lohn einlassen müssen, bevor sie nachträglich ggf. zu einer faireren Bezahlung gelangen, kann sie erst nach Vertragsschluss oder Beendigung des Arbeitsverhältnisses wirken. Oft bestehen Loyalitätskonflikte und die Angst um den Verlust des Arbeitsplatzes (vgl. Franke (Fn. 65), S. 256). Hinzu kommt die organisatorische Unterlegenheit von Individuen gegenüber Unternehmen (vgl. Pfarr/Kocher, NZA 1999, 358 f.). Daher wird zur Effektivierung der Rechtsdurchsetzung ein Verbandsklagerecht der Gewerkschaften gefordert (dazu näher: Bepler (Fn. 35), S. 203; Peter (Fn. 5), S. 124 f.; Pfarr/Kocher, NZA 1999, 358/364). 